\title{
Etograma de gaviões-carijós (Rupornis magnirostris) internados no ambulatório de animais selvagens da Universidade Federal Rural da Amazônia
}

Ethogram of carijós hawks (Rupornis magnirostris) hospitalized in the wildlife outpatient clinic of

\author{
the Universidade Federal Rural da Amazônia
}

Ethograma de halcones carijós (Rupornis magnirostris) hospitalizados en la clínica ambulatoria de vida silvestre de la Universidade Federal Rural da Amazônia

Recebido: 25/03/2021 | Revisado: 30/03/2021 | Aceito: 03/04/2021 | Publicado: 04/05/2021

Alexsandro Antônio Portilho Damasceno
ORCID: https://orcid.org/0000-0002-5848-9721
Universidade Federal Rural da Amazônia, Brasil
E-mail: antonioalex.damasceno7@gmail.com
João Vitor Da Silva Oliveira
ORCID: https://orcid.org/0000-0003-2680-7534
Universidade Federal Rural da Amazônia, Brasil
E-mail: jvoliveira906@ @mail.com
Mariana Jucá Moraes
ORCID: https://orcid.org/0000-0002-1175-177X
Universidade Federal Rural da Amazônia, Brasil
E-mail: marianajmoraes15@gmail.com
Juliana Maria Santos Miranda
ORCID: https://orcid.org/0000-0003-4608-8653
Universidade Federal Rural da Amazônia, Brasil
E-mail: juhmiranda30@gmail.com
Danniel De Carvalho Castro
ORCID: https://orcid.org/0000-0002-1705-5054
Universidade Federal Rural da Amazônia, Brasil
E-mail: danniel.hg @gmail.com
Cecília Casimiro Do Carmo
ORCID: https://orcid.org/0000-0001-7462-1887
Universidade Federal Rural da Amazônia, Brasil
E-mail: cee_ciliia @hotmail.com
Ana Sílvia Sardinha Ribeiro

\section{Resumo}

As aves de rapina são aves predadoras, exuberantes e estão presentes em todos os estados do Brasil, especialmente o Rupornis magnirostris também conhecido como gavião-carijó, pega pinto. Esta ave possui uma alimentação bastante diversificada e também apresenta um comportamento extremante territorialista, haja vista que quando retirados da natureza e criados em cativeiro, eles alteram seu comportamento para que sejam capazes de se adaptar ao novo meio. Dessa forma, o presente trabalho tem como objetivo a elaboração de etograma de Rupornis magnirostris cativos a fim de acrescentar mais informações disponíveis sobre a biologia e comportamento desta espécie em cativeiro. Para isto, foi executado um etograma utilizando a técnica ad libitum realizada em 3 turnos diários com duração de 30 minutos cada para identificação dos comportamentos, e posteriormente foi utilizada a técnica animal-focal dividida em 3 turnos, com duração de 30 minutos realizada em 21 sessões, durante 10 dias, obtendo-se como resultado o comportamento posição neutra o mais expressado, resultado da falta de enriquecimento ambiental e estímulo. Portanto, as condições de ambiência e física dos gaviões são de extrema importância para compreender o comportamento desta espécie em cativeiro.

Palavras-chave: Etologia; Comportamento; Aves de rapina; Cativeiro.

\section{Abstract}

Birds of prey are predatory birds are present in all states of Brazil, especially of Rupornis magnirostris. This bird has a very diverse diet presents an extremely territorial behavior, respecting when are withdraw from nature and raised in captivity, they are change their behavior so that they are able to adapt to the new middle. In this way, this study has the objective the elaboration of the ethogram of Rupornis magnirostris captive in order to add more information 
available on the biology and behavior of this species in captivity. For this, an ethogram was performed using the ad libitum technique performed in 3 shifts lasting 30 minutes each and after this, the Focal-Animal technique was used, divided into 3 shifts, lasting 30 minutes, performed in 21 sessions, during 10 days. Obtaining as a result the neutral position behavior the most expressed result of the lack of environmental enrichment and stimulus. Therefore, the ambience and physical conditions of the hawks are extremely important to understand the behavior of this species in captivity.

Keywords: Behavior; Birds of prey; Captivity; Etology.

\section{Resumen}

Las aves rapaces son aves depredadoras, exuberantes y están presentes en todos los estados de Brasil, especialmente Rupornis magnirostris también conocido como halcón-carijó, captura pinto. Esta ave tiene una dieta muy diversa y también presenta un comportamiento territorialista extremo, dado que cuando se toma de la naturaleza y se cría en cautiverio, alteran su comportamiento para que sean capaces de adaptarse al nuevo entorno. Así, el presente trabajo tiene como objetivo elaborar ethograma de Rupornis magnirostris cautivos con el fin de añadir más información disponible sobre la biología y el comportamiento de esta especie en cautiverio. Para ello, se realizó un ethograma utilizando la técnica ad libitum realizada en 3 turnos diarios que duraban 30 minutos cada uno para identificar los comportamientos, y posteriormente se utilizó la técnica animal-focal dividida en 3 turnos, con una duración de 30 minutos en 21 sesiones, durante 10 días, lo que dio lugar a la posición neutral más expresada, resultante de la falta de enriquecimiento ambiental y estímulo. Por lo tanto, las condiciones de ambiente y física de los halcones son extremadamente importantes para entender el comportamiento de esta especie en cautiverio.

Palabras clave: Etología; Comportamento; Laves de presa; Cactivador.

\section{Introdução}

O aumento exacerbado da ação antrópica no ambiente vem promovendo alterações nos biomas do país, dentre elas a perda e a fragmentação de habitat de vários grupos animais, a exemplo do das aves (Silva, 2016).

O processo de ocupação de animais silvestres em ambientes urbanos é esperado, tendo em vista a fragmentação dos ecossistemas florestais, os centros urbanos são uma alternativa natural considerando a fartura de alimentos e que até mesmo do lixo alguns desses animais conseguem tirar seu alimento.

A variabilidade de localizações com possibilidade para buscar abrigo, torna o ambiente urbano um novo habitat desses animais resultando em conflitos com o homem, levando a fauna silvestre a se tornar vítima de ações antrópicas como poluição sonora, violência decorrendo do desconhecimento da importância ecológica e própria a mistificação dos riscos de um animal da fauna selvagem (Vilela, 2016), pensamentos como toda a cobra é venenosa, todos os morcegos possuem raiva e a recente que primatas são vetores da febre amarela. Esse contato da fauna selvagem com a população urbana leva à um aumento no número de resgates em centros urbanos e atendimentos em hospitais veterinários, como o Hospital Veterinário Mário Dias Teixeira da UFRA Belém e o Ambulatório de Animais Selvagens detendo a importante missão da manutenção da biodiversidade considerando a falta de conhecimento prévio da população.

Entre as espécies mais capturadas no ambiente urbano, destacamos as aves rapinantes, que sofrem ações antrópicas diretas e indiretas, como os acidentes por arma de fogo, baladeiras, linhas de cerol, queda do ninho, entre outras causas. Destes, ressaltamos o Rupornis magnirostris (gavião-carijó), espécie rotineiramente encaminhada pelos órgãos ambientais, o gaviãocarijó é uma espécie do grupo das aves de rapina presente em todo o Brasil, ocorrendo desde norte do México, América Central até a Argentina, Peru e é um animal com ótima adaptação aos impactos ambientais e facilmente encontrado nas áreas urbanas (Santos \& Rosado, 2009), apresentando uma grande variação de cores na plumagem, conforme a região do país. Em qualquer uma, no entanto, destaca-se o peito finamente barrado da barriga e a cauda com várias faixas claras em contraste com as faixas cinza escuras ou negras (Antas, 2005).

Segundo Tortato (2009), essas aves apresentam um hábito alimentar de típico caçador oportunista, constituída de grandes insetos, alguns répteis, anfíbios, pequenas cobras e pássaros, tais como rolas (Zenaida auriculata) e pardais (Passer domesticus). A necessidade de alimento diário destas aves varia de acordo com a estação climática e com o seu tamanho 
corpóreo (Granzinilli, 2003). Essa ave tem um papel ecológico importante, por ser um predador de topo, é responsável pela manutenção e funcionamento de comunidades as quais pertencem (Rickefs, Miller, 2000).

Referente ao comportamento da ave é um rapinante que vive solitário ou aos pares, passando a maior parte do tempo empoleirado no alto de árvores como as outras aves de rapina (Ferguson-lees \& Christie, 2001), além disso é uma ave extremamente territorial que anuncia a presença vocalizando e circulando em voos altos, aproveitando as correntes de ar quente (Sick, 1997) e esse grito é um alerta característico da espécie, usado como defesa e é emitido assim que qualquer intruso chega em seu território (Antas, 2005).

Ao se retirar um animal selvagem do seu ambiente natural há o estresse inerente provocado pela situação, esse estado de perturbação torna-se um dos elementos prejudicial capacidade dos indivíduos de se adaptarem ao novo meio (Broom, 1986), ou seja, quando são criados em cativeiro, os animais alteram seu comportamento como uma forma de adaptação à sua nova realidade (Albuquerque, Silveira \& Oliveira, 2009), dessa forma, o etograma é uma ferramenta básica para observação da ecologia e biologia de animais em cativeiro ou em vida livre (Alcook, 1997), esse por sua vez tem como característica listar os comportamentos das espécies, e por meio disso reconhecer e estudar os comportamentos do animal e correlacionar com diversos fatores ambientais (Souto, 2005).

Com à carência de informações sobre o comportamento da espécie Rupornis magnirostris e também com objetivo de aumentar os conhecimentos sobre a mesma, o trabalho consistiu na elaboração de etograma quantitativo de gaviões-carijó em cativeiro, visando acrescentar informações disponíveis sobre a biologia e comportamento desta espécie.

\section{Metodologia}

A pesquisa foi realizada no Ambulatório de Animais Selvagens do Hospital Veterinário Mário Teixeira Dias, localizado na Universidade Federal Rural da Amazônia- UFRA e os procedimentos realizados estavam de acordo com a Comissão de Ética no Uso de Animais (CEUA) da UFRA sob o registro 026/2018 e 23084.0158852/2018-51.

Foram utilizados quatro gaviões-carijós adultos, sem sexo definido, recebidos no período de 2016 a 2018, todos com a procedência do Batalhão de Polícia Ambiental (PA). Estes foram denominados de Carijó 1 a 4 e distribuídos em 4 recintos, os quais eram feitos de ferro com dimensões $50 \mathrm{~cm} \times 60 \mathrm{~cm} \times 30 \mathrm{~cm}$ contendo apenas um único poleiro de madeira, esses ficavam um próximo dos outros para que os animais pudessem se ver e interagir entre si. A alimentação de todos os animais era composta por $35 \mathrm{~g}$ moela de frango suplementada com $0,15 \mathrm{ml}$ de cálcio.

\subsection{Etograma}

O estudo comportamental baseou-se de acordo com as técnicas ad libitum e animal-focal de Altiman (1974), a primeira foi realizada com a finalidade da identificação dos comportamentos, foram consistindo em 3 turnos de duração de 30 minutos cada um. E segunda técnica que também foi utilizada, a técnica de animal-focal, onde todas as atividades individuais ou interações entre os animais são contabilizadas. A coleta dos dados foi executada em 3 turnos, com duração de 30 minutos cada, que foram fracionados em 21 sessões diárias, durante o período de 10 dias. As sessões focais duravam 30 minutos que por sua vez eram divididos em observações individuais de 5 minutos para cada animal observado, com 5 minutos de intervalo entre sessões. A primeira sessão era de 8:00 às 8:30, a segunda de 12:00 às 12:30 e a última sessão era de 16:00 às 16:30 horas.

Durante as observações não existiam quaisquer interação entre os animais e observador e não coincidiam com o horário de limpeza dos recintos, mas coincidiam com os horários de alimentação e eram feitas a uma distância de $2 \mathrm{~m}$ do recinto das aves e os comportamentos observados eram registrados em quadro pré-estabelecido contendo os comportamentos esperados pela espécie e posteriormente após o termino de cada sessão eram registrados em planilhas, onde foi feita análise dos dados comportamentais por turno e quais eram mais eram realizados. Foram registrados 15 comportamentos executados 
pelas aves. É importante enfatizar que a análise dos dados será quantitativa, onde a expressão comportamental dos animais é coletada de forma objetiva após a observação durante um período amostral variável, segundo Rapp (2007).

\section{Resultados e Discussão}

Tabela 1. Somatório das frequências absolutas dos comportamentos observados.

\begin{tabular}{cccccc}
\hline & $\begin{array}{c}\text { Somatório do } \\
\text { primeiro } \\
\text { horário }\end{array}$ & $\begin{array}{c}\text { Somatório do } \\
\text { segundo } \\
\text { horário }\end{array}$ & $\begin{array}{c}\text { Somatório do } \\
\text { terceiro } \\
\text { horário }\end{array}$ & $\begin{array}{c}\text { Total } \\
\text { absoluto }\end{array}$ & $\begin{array}{c}\text { Total } \\
\text { relativo \% }\end{array}$ \\
\hline Posição Neutra & 167 & 108 & 165 & 440 & $53 \%$ \\
\hline Andar & 23 & 19 & 20 & 62 & $7 \%$ \\
\hline Saltar & 20 & 13 & 10 & 43 & $5 \%$ \\
\hline Sacudir o rabo & 3 & 1 & 0 & 4 & $0 \%$ \\
\hline Repousar patas & 7 & 3 & 3 & 13 & $2 \%$ \\
\hline Ataque físico & 10 & 14 & 22 & 46 & $6 \%$ \\
\hline Estado de alerta & 25 & 40 & 33 & 98 & $12 \%$ \\
\hline Sacudir pernas & 3 & 1 & 17 & 41 & $5 \%$ \\
\hline Limpar penas & 18 & 6 & 0 & 2 & $0 \%$ \\
\hline Bocejar & 1 & 1 & 0 & 64 & $8 \%$ \\
\hline Comer & 0 & 64 & 1 & 6 & $1 \%$ \\
\hline Vocalização & 0 & 5 & 2 & 3 & $0 \%$ \\
\hline Sacudir cabeça & 0 & 1 & 1 & 2 & $0 \%$ \\
\hline Defecar & 0 & 1 & 277 & 831 & $100 \%$ \\
\hline Total & 277 & & & & $5 \%$ \\
\hline
\end{tabular}

Fonte: Autores.

O comportamento de maior frequência no primeiro intervalo de observações, que foi das 8:00 às 8:30 horas foi a "posição neutra", com 60\% de frequência, similar ao que foi encontrado por (Oliveira; Souza; Silva, 2014), no etograma de Carcarás (Caracara Plancus), (Fabiano, 2017) com Tucanos-toco (Ramphatos toco) e Herculano; Santos; Pigozzo, (2013) com o etograma de Flamingo-chileno (phoenicopteriformes, phoenicopteridae), que obtiveram 60,46\% no comportamento “descanso", 55,66\% na categoria "inatividade" e 57,70\% na categoria manutenção, respectivamente, sendo que os trabalhos citados recolheram os comportamentos e os agrupou em categorias. Este trabalho está apresentando o resultado das observações sem o uso de grupos comportamentais, apenas pela frequência absoluta de cada comportamento. A frequência de $60 \%$ do comportamento posição neutra pode ser explicada pelo ambiente cativo carente de enriquecimento ambiental, resultando-nos animais inativos e em ociosidade (Assis et al., 2016).

Em relação ao horário de atividade dos animais, apesar de Medeiros (2015) indicar que os carijós são predadores tanto diurnos como noturnos, neste estudo não houve coleta de dados no horário noturno para comparar com esta afirmação, porém, no final da tarde quando ocorreu a terceira observação, a porcentagem foi mantida, dessa forma concordando com o estudo de Medeiros de forma parcial, no entanto é necessário mais estudos sobre o comportamento dessa espécie, outro fator que se especula para a justificativa da inatividade das aves é ausência de pessoas no período mais cedo de observação, o silêncio e falta de estímulos ambientais resulta na ociosidade dos animais. Entretanto, no etograma do Flamingo-chileno (Phoenicopterus chilensis) de Herculano e Santos; Pigozzo, (2013) da conduta de alerta das aves estavam relacionadas em resposta a fortes ruídos, como a aproximação de um helicóptero e como também, com o momento de entrada do tratador para trazer o alimento ou limpar no recinto, isto, também é fator que leva a falta conduta de alerta e a presença da ociosidade pelos gaviões.

O comportamento limpar penas apresentou apenas 7\% de frequência no trabalho apresentado, no entanto, no estudo de Oliveira, Souza e Silva (2014) analisando o comportamento do Caracara plancus esta atividade apresentou maior número 
de repetições, tal diferença pode ser pelo tamanho dos recintos que os Carcarás e os Carijós usufruíam, pois o fato das gaiolas dos Rupornis magnirostris serem menores promove a diminuição da expressão de vários comportamentos naturais da espécie (Alves et al., 2007) (Figura 1).

Figura 1. Comportamentos de todos os gaviões no horário de 8:00-8:30.

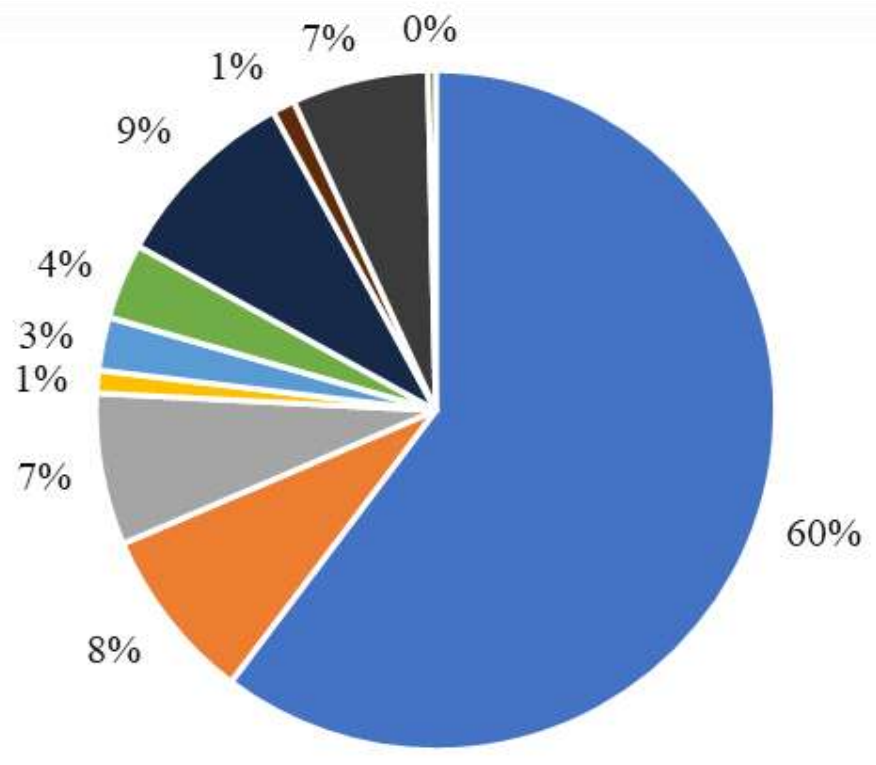

- Posição Neutra

- Andar

= Saltar

- Sacudir o rabo

- Repousar patas

- Ataque físico

- Estado de alerta

- Sacudir pernas

- Limpar penas

- Bocejar

Fonte: Autores.

No segundo intervalo de observações, que consistiu no horário de 12:00 as 12:30 horas, o comportamento observado com maior frequência foi a "posição neutra" com $40 \%$ de frequência em consequência da particularidade do carijó 1 , seguido do comportamento "comer" com $23 \%$ de frequência, visto que foi este o horário em que foi ofertado a alimentação dos rapinantes e como já discutido, a existência de uma atividade qualquer como a alimentação diminuiu o tempo de ociosidade dos animais de forma considerável demonstrando a importância do enriquecimento ambiental, pois a aplicação dessas técnicas reduzem o tempo ocioso de animais cativos, por assemelhar o ambiente de cativeiro ao ambiente natural, aumentando seu bem estar (Assis et al., 2016). Como também foi possível observar com 15\% de frequência, o comportamento "estado de alerta", pois todos os animais do local estavam sendo alimentados no mesmo horário, tendo em vista que as gaiolas estavam lado-alado e o que o Gavião-carijó é extremamente territorial (Sick, 1997), resultava na tensão entre as aves no momento da alimentação (Figura 2). 
Figura 2. Comportamento de todos os gaviões no horário de 12:00-12:30.

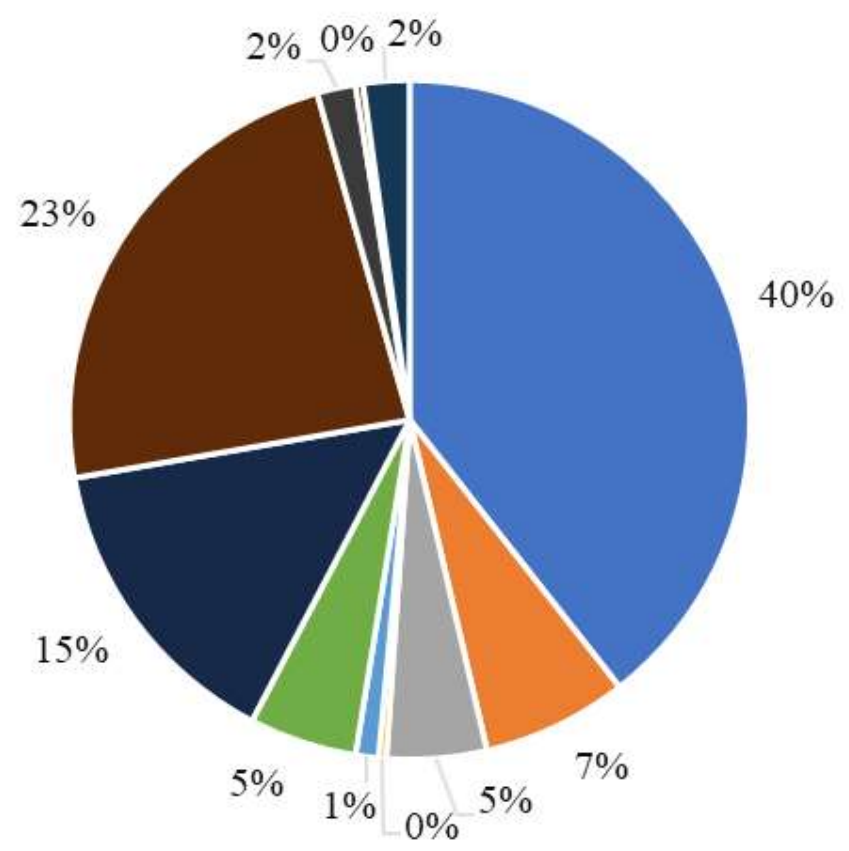

- Posição Neutra

- Andar

- Saltar

" Sacudir o rabo

- Repousar patas

- Ataque físico

- Estado de alerta

- Comer

- Vocalização

- Sacudir pemas

- Limpar pernas

Fonte: Autores.

Os comportamentos dos gaviões-carijós foram comparados com de outros animais, como no trabalho de Henrique e Piratelli (2008), com Garça-Branca Grande (Casmerodius albus, Linnaeus, 1758) de vida livre, mostrou que essas aves passaram $27 \%$ do tempo alimentando-se e $18 \%$ do tempo locomovendo-se dentro do ambiente, no trabalho devido a busca pelo alimento do animal, por outro lado em cativeiro devido ao fato dos gaviões-carijós receberem o alimento sem necessidade nenhuma de caçar ou ter muito esforço para dilacerar o alimento fornecido, isto pode explicar o fato de passar maior parte do tempo em posição neutra no horário período de 12:00 ás 12:30, e como também a frequência de $23 \%$ do comportamento “comer”e 7\% “ andar”, no trabalho do Flamingo-chileno (Herculano; Santos; Pigozzo, 2013) a categoria alimentação chegou aos $17,40 \%$ e a categoria locomoção chegou aos $6,10 \%$, resultados bem próximos aos encontrados com o comportamentos dos Carijós.

No último intervalo de observação, no horário de 16:00 às 16:30, o comportamento mais observado foi a "posição neutra" com $60 \%$ de frequência, seguido do "estado de alerta" com $12 \%$ de frequência. Outros dois comportamentos que foram observados nesse horário, porém com pouca frequência foram os comportamentos "andando" e "limpando penas", com 7\% e $6 \%$ de frequência, respectivamente (Figura 3). 
Figura 3. Comportamento de todos os gaviões no horário de 16:00-16:30.

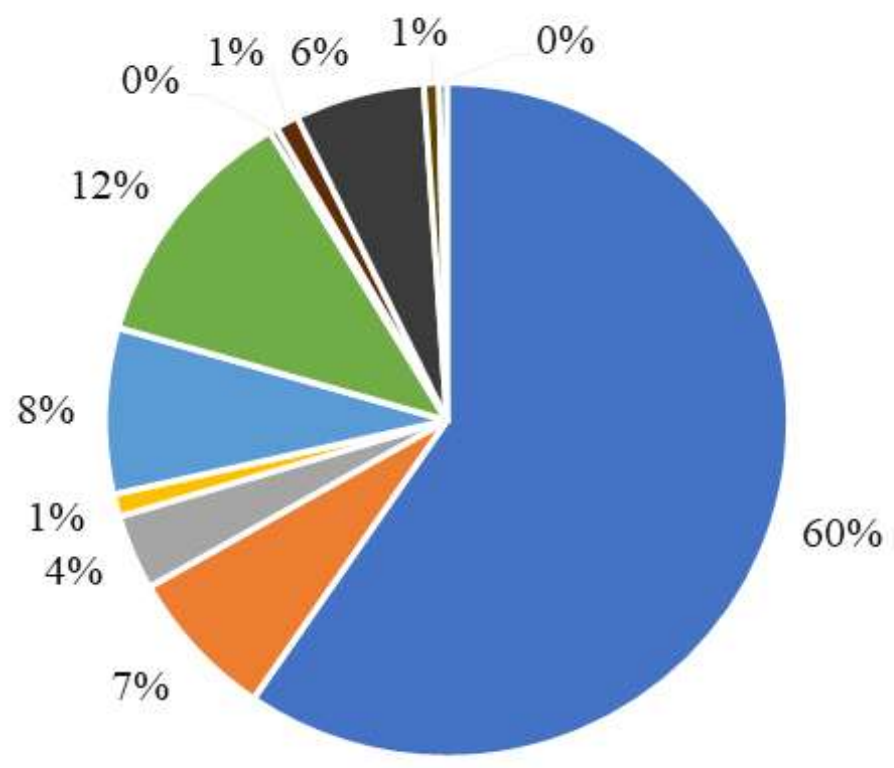

- Posição Neutra

- Andar

$=$ Saltar

" Repousar patas

- Ataque físico

- Estado de alerta

- Vocalização

- Sacudir pernas

- Limpar pernas

- Sacudir cabeça

- Defecar

Fonte: Autores.

Comparando o resultado obtido no gráfico acima com o trabalho de Henrique e Piratelli (2008) em seu estudo com Garça-branca-grande (Casmerodius albus), que concluíram que o tempo empregado para locomoção, $(\mathrm{n}=37,8$ horas; $18 \%$ ) foi considerado pequeno. O comportamento "andando", em Rupornis magnirostris com 7\% de frequência, se encaixa na categoria de locomoção. Este comportamento foi realizado em menor frequência comparado ao trabalho de Casmerodius albus.

Os resultados obtidos nesse trabalho mostram uma parte significativa do estudo comportamental de Rupornis magnirostris criados em cativeiro, o que pode não só aprimorar o conhecimento sobre a biologia e comportamento da espécie, mas também auxiliar em melhorias no seu manejo e consequentemente proporcionar melhores condições de bem estar para esses animais em cativeiro, a partir da compreensão dos seus comportamentos. Assim como o Etograma do Carcará (Caracara plancus, Miller, 1777), em cativeiro, Oliveira, Souza e Silva (2014) que foi o primeiro etograma a analisar o comportamento do Carcará em cativeiro, este também é o primeiro etograma que analisa os comportamentos de Rupornis magnirostris em cativeiro. Portanto, é necessário ampliar de estudos sobre comportamento da espécie para poder relacionar os resultados observados nesse trabalho com os comportamentos de R. magnirostris não cativos.

\section{Conclusão}

Concluímos que com as condições de ambiência do local, os animais passaram a maior parte do tempo em posição neutra, por conta do recinto em que vivem não ser o local ideal e pelas condições de saúde de dois desses animais observados, uma melhoria na ambiência desses animais e a promoção de enriquecimentos ambientais poderia aprimorar e proporcionar mais bem estar à estadia dos internados. Recomenda-se, ainda a realização estudos em cativeiro com gaiolas ou recintos maiores, os quais amenizariam as limitações na expressão de comportamentos inerentes do cativeiro, e a observação dos animais por meio do uso de câmeras digitais, as quais permitiriam a observação durante as 24 horas do dia as quais eliminariam qualquer tipo de interferência humana e além disso forneceriam uma amostra comportamental maior em dados quantitativos e qualitativos, para assim poder descrever melhor esses comportamentos e elaborar até catálogos contendo 
ilustrações de cada comportamento expressado pelas aves em cativeiro, como também a realização de estudos similares com as aves em vida livre.

\section{Agradecimentos}

Agradecemos ao ambulatório de Animais Selvagens, anexo ao hospital veterinário/HOVET da Universidade Federal Rural da Amazônia por nós dá o suporte e permitir a realização da presente pesquisa.

\section{Referências}

Alcock, J. (1997). Animal behavior; anevolutionaryapproach (3a ed.). Sinauer Associates.

Altimann, J. (1974). Observational study of behavior: sampling methodos. Behaviour, 49(3), 227-267.

Alves, S. P., Silva, I. J. O., \& Piedade, S. M. S. (2007). Avaliação do bem-estar de aves poedeiras comerciais: efeitos do sistema de criação e do ambiente bioclimático sobre o desempenho das aves e a qualidade dos ovos. Revista Brasileira de Zootecnia, 36, 1388 -1394.

Antas, P. T. Z. (2005) Aves do Pantanal. RPPN: Sesc. http://www.avesderapinabrasil.com

Assis, V. D. L., Carvalho, T. S. G., Pereira, V. M., Freitas, R. T. F., Saad, C. E. P., Costa, A. C. \& Silva, A. A. A. (2016). Environmental enrichment on the behavior and welfare of cockatiels (Nymphicushollandicus). Arquivo Brasileiro de Medicina Veterinária e Zootecnia, 68, $562-570$.

Broom, D. M. (1986) Indicators of poor welfare. British Veterinary Journal, 142, 524-526.

Fabiano, K. L. N. (2017). Avaliação de técnicas de enriquecimento ambiental aplicadas à Ramphastos toco (Statius Muller, 1776) (Aves, Ramphastidae), em cativeiro. Monografia (Bacharel em Ciências Biologicas), Universidade Federal de Uberlândia.

Ferguson-Less, J. \& Christies, D. A. (2001) Raptorsofthe world. HoughtonMifflinCompany.

Granzinolli, M. A. M. (2003). Ecologia Alimentar do gavião-do-rabo-branco Buteo aubicaudatus (Falconiformes:Accipitridae) no município de Juiz de Fora, sudeste do estado de Minas Gerais. Dissertação (Mestre em Biociência), Instituto de Biociências da Universidade de São Paulo.

Henrique, C. A. M., \& Piratelli, A. (2008). Etograma da garça-branca-grande, Casmerodiusalbus (Ciconiiformes, Ardeidae). RevistaBrasileira de Ornitologia, p.185-192.

Herculano, D. M., Santos, M. A. B., \& Pigozzo, C. M. (2013). Etograma de Flamingo Chileno, Phoenicopteruschilensis (phoenicopteriformes, phoenicopteridae), em condição de cativeiro no Parque Zoobotânico Getúlio Vargas. Candombá Unijorge. 9(1) 08-21.

Medeiros, B. F. (2015). Análise histológica do trato intestinal do gavião-carijó (Rupornismagnirostris, Gmelin, 1877). Monografia (Bacharel em Ciências Biologicas). Vitória de Santo Antão.

Oliveira, H. S., Souza, D. R. A., \& Silva, M. N. (2014). Etograma do Carcará (Caracara plancus, Miller, 1777) (Aves, Falconidae), em cativeiro. Revista de Etologia, 13(2) 1-9.

Rapp P. E. (2007). Quantitative characterization of animal behavior following blast exposure. Cognitive neurodynamics, 1(4), 287-293. https://doi.org/10.1007/s11571-007-9027-8

Ricklefs, R. E., \& Miller, G. L. (200). Ecology. W. H. Freeman and Company, USA.

Santos, W. M., \& Rosado, F.R. (2009). Dados preliminares da biologia do Gavião-carijó (Rupornis magnirostris, gmelin, 1788) na Região Noroeste do Paraná. Revista em Agronegócios e Meio Ambiente, 2 (3) 421-430.

Sick, H. (1997). Ornitologia brasileira. Editora Nova Fronteira.

Silva, E. F. A. (2016). Análise microbiológica e morfológica do trato intestinal de gaviões-carijós (Rupornis magnirostris GMELIN, 1788) provenientes do CETAS-IBAMA/PE. Dissertação (Mestre em Saúde Humana e Meio Ambiente), Disponível em: https://repositorio.ufpe.br/handle/123456789/17319

Souto, A. 2005. Etologia: princípios e reflexões (3a ed.), UFPE.

Tortato, M. A. (2009). Predação de cuíca-d’água (Chironectes minimus: Mammalia, Didelphidae) por gavião-carijó (Rupornis magnirostris: Aves, Accipitridae). Mastozoología Neotropical, 16 (2) 491-493.

Vilela, D. A. R., Teixeira, C. P., Horta, C. C. R., Loura, G. R., \& Silva, M. M. (2016). Gestão de conflitos com animais silvestre em centros urbanos. 60p. 